Published in final edited form as:

Ann Neurol. 2018 March ; 83(3): 575-587. doi:10.1002/ana.25180.

\title{
Stereotactic Laser Amygdalohippocampotomy for Mesial Temporal Lobe Epilepsy
}

\author{
Robert E. Gross, MD, PhD ${ }^{1,2}$, Matthew A. Stern, BS ${ }^{3}$, Jon T. Willie, MD, PhD ${ }^{1,2}$, Rebecca E. \\ Fasano, MD $^{2}$, Amit M. Saindane, MD $^{4}$, Bruno P. Soares, MD $^{6}$, Nigel P. Pedersen, MBBS ${ }^{2}$, \\ and Daniel L. Drane, PhD $2,5,6$ \\ ${ }^{1}$ Department of Neurosurgery, Emory University School of Medicine \\ ${ }^{2}$ Department of Neurology, Emory University School of Medicine \\ ${ }^{3}$ Medical Scientist Training Program, Emory University School of Medicine \\ ${ }^{4}$ Department of Radiology and Imaging Sciences, Emory University School of Medicine \\ ${ }^{5}$ Department of Pediatrics, Emory University School of Medicine \\ ${ }^{6}$ Department of Neurology, University of Washington School of Medicine
}

\begin{abstract}
Objective-To evaluate the outcomes one-year and longer following stereotactic laser amygdalohippocampotomy for mesial temporal lobe epilepsy in a large series of patients treated over a five-year period since introduction of this novel technique.

Methods-Surgical outcomes of a consecutive series of fifty-eight patients with mesial temporal lobe epilepsy who underwent the surgery at our institution with at least 12-months follow-up were retrospectively evaluated. A subgroup analysis was performed comparing patients with and without mesial temporal sclerosis.
\end{abstract}

Corresponding Author: Robert E. Gross, MD PhD, Department of Neurosurgery, Emory University School of Medicine, 1365 Clifton Road NE, Suite B6200, Atlanta, GA 30322, Phone: 404-727-2354, Fax: 404-712-8576, rgross@emory.edu; Daniel L. Drane, $\mathrm{PhD}$, Departments of Neurology and Pediatrics, Emory University School of Medicine, 1365 Clifton Road NE, Suite 6111, Atlanta, GA 30322, Phone: 404-727-2844, ddrane@emory.edu.

Author Contributions

RG, MS, JW and DD contributed to the conception and/or design of the study; RG, MS, JW, RF, AS, BS, NP and DD contributed to the acquisition and/or analysis of the data; RG, MS, JW, AS, BP and DD contributed to the drafting of the text and/or preparation of the figures.

Potential Conflicts of Interest

RG: Dr. Gross has served as a consultant to Medtronic, Monteris, MRI interventions, Visualase, Inc., and Zimmer Biomet, which manufacture products related to the research described in this paper and may be affected by this study, and receives compensation for these services. Dr. Gross received research support from Medtronic, Inc. as part of the SLATE trial (their multisite prospective trial evaluating SLAH), and has received research grants from Visualase, Inc., and MRI Interventions. The terms of these arrangements have been reviewed and approved by Emory University in accordance with its conflict of interest policies.

JW: Dr. Willie has served as a consultant to Medtronic, Inc. and MRI Interventions, Inc., and has received compensation for these services, and receives research support from Medtronic, Inc. as part of the SLATE trial. The terms of these arrangements have been reviewed and approved by Emory University in accordance with its conflict of interest policies.

RF: Dr. Fasano receives research support from Medtronic, Inc. for the SLATE trial.

DD: Dr. Drane through Emory University has received a research grant from Medtronic, Inc., and currently serves as the core lab director for their SLATE trial. The terms of these arrangements have been reviewed and approved by Emory University in accordance with its conflict of interest policies.

Authors MS, AM, BS and NP have nothing to report. 
Results-One-year following stereotactic laser amygdalohippocampotomy 53.4\% (95\% confidence interval: $40.8 \%-65.7 \%$ ) of all patients were free of disabling seizures (Engel 1). Three of nine patients became seizure free following repeat ablation. Subgroup analysis showed that $60.5 \%$ (95\% confidence interval: $45.6 \%-73.7 \%$ ) of patients with mesial temporal sclerosis were free of disabling seizures as compared to $33.3 \%$ (95\% confidence interval: $15.0 \%-58.5 \%$ ) of patients without mesial temporal sclerosis. Quality of Life in Epilepsy-31 scores significantly improved at the group level, few procedure-related complications were observed, and verbal memory outcome was better than historical open resection data.

Interpretation-In an unselected consecutive series of patients, stereotactic laser amygdalohippocampotomy yielded seizure-free rates for patients with mesial temporal lobe epilepsy lower than, but comparable to, the outcomes typically associated with open temporal lobe surgery. Analogous to results from open surgery, patients without mesial temporal sclerosis fared less well. This novel procedure is an effective minimally invasive alternative to resective surgery. In the minority of patients not free of disabling seizures, laser ablation presents no barrier to additional open surgery.

\section{Introduction}

Mesial temporal lobe epilepsy (MTLE) represents roughly one quarter of all cases of epilepsy, approximately one-third of which are refractory to medication ${ }^{1}$. The standard surgical treatment of drug-resistant MTLE has been open surgical resection of the medial temporal lobe, including most of the hippocampus and amygdala as well as the parahippocampal gyrus. This can be performed with an anterior temporal lobectomy (ATL) ${ }^{2}$ or via a more 'selective' approach (selective amygdgalohippocampectomy, SAH) which avoids resection of the anterior temporal lobe, gaining access to the medial temporal lobe by transection through a white matter corridor. A recent meta-analysis comparing ATL and SAH demonstrated one-year rates of freedom from disabling seizures of $75 \%$ and $67 \%$, respectively (determined from the reported risk ratios and absolute risk reductions) ${ }^{3}$. The analysis showed that 13 patients needed to be treated to render one additional patient seizure free after ATL.

Anterior temporal lobectomy has been associated with cognitive impairments and focal neurological deficits ${ }^{4-10}$, particularly with regard to verbal memory ${ }^{11}$ and aspects of naming ability ${ }^{8-10}$. The rationale to perform a more selective resection (i.e. SAH) has been, at least in part, to minimize neurocognitive impact related to resection of the anterior temporal lobe. However, SAH has not clearly prevented cognitive declines ${ }^{7-9,}$ 12-14. In fact, on the dominant side, SAH has been associated with greater declines in verbal learning than ATL, which has been related to the degree of 'collateral damage' within the temporal stem resulting from its transgression during the approach to the medial temporal structures. ${ }^{15}$ Novel surgical approaches that minimize the degree of collateral injury during ablation of medial temporal structures may reduce cognitive impact, but perhaps at a cost to seizure outcome. That tradeoff may determine the relative benefits of such a minimally invasive approach. 
Stereotactic laser amygdalohippocampotomy (SLAH) utilizes minimally-invasive stereotactic access for insertion of a cooled catheter through which an optical fiber delivers laser energy. Laser heating, controlled by real time MR-guided thermal mapping, produces thermocoagulative necrosis of the hippocampus, subiculum, amygdala, and uncus, while relatively sparing other medial, basal and lateral temporal structures. ${ }^{16,17}$ In early studies, SLAH has shown promise for stopping seizures while avoiding neurocognitive declines typically associated with ATL and SAH. ${ }^{18}$ To better determine the safety and effectiveness of this procedure, we report outcomes at one-year following SLAH in 58 patients with MTLE, with or without mesial temporal sclerosis (MTS), treated over the first five years after the introduction of the laser ablation for epilepsy.

\section{Methods}

\section{Patient Selection}

All patients who underwent SLAH for MTLE between July 1, 2011 and June 30, 2016 were included in this retrospective analysis, which was performed under a protocol approved by the Emory University Institutional Review Board. Candidates for surgical treatment underwent a standard diagnostic epilepsy evaluation comprised of long-term inpatient scalp video electroencephalographic monitoring (vEEG), 3T (unless contraindicated) MRI including coronal T2 and T2-fluid-attenuated inversion recovery (FLAIR) sequences, 18fluorodeoxyglucose positron emission tomography $\left({ }^{18}\right.$ FDG-PET) and neuropsychological testing. The pre-operative MRI scans were evaluated by neuroradiologists and neurosurgeons for presence of MTS, defined as atrophy of the hippocampus with loss of internal architecture and/or presence of increased signal on T2 and/or T2-FLAIR sequences. If necessary, additional testing was performed, including language lateralization determination via functional MRI; intracarotid amobarbital (Wada) testing; and/or invasive EEG monitoring via depth, strip, grid and/or foramen ovale electrodes. Final vetting for surgery was determined in a multidisciplinary conference that included epileptologists, neurosurgeons, neuroradiologists, and neuropsychologists. Patients who had electrographic evidence of unilateral anterior temporal onsets on scalp EEG and/or medial temporal onsets on invasive EEG, with concordant MTS, if present, and/or concordant temporal hypometabolism on PET, were offered a choice of open resection or SLAH, although later in the series the option of open temporal surgery was not offered to patients with dominant hemisphere onsets.

\section{Surgical procedure}

One of two surgeons (REG and JTW) performed SLAH with patients under general anesthesia using the Visualase ${ }^{\circledR}$ system (Medtronic Inc., Lewiston, CO) for laser energy delivery and magnetic resonance thermal imaging, as previously described ${ }^{19-21}$. Some patients underwent implantation of the laser catheter in the operating room using a standard stereotactic head frame (CRW; Integra Neurosciences, Plainsboro, NJ), but the majority of patients underwent implantation of the catheter in the MRI scanner using a MRI-guided trajectory frame (ClearPoint ${ }^{\circledR}$ ScalpMount SmartFrame, MRI Interventions, Irvine, CA). Trajectories were chosen to penetrate the central portion of the hippocampus from the body at the level of the tectal plate through the head, continuing through the amygdala to the 
medial temporal pole. The laser fiber was inserted and adjusted if the location was suboptimal. Thermal maps were acquired continuously throughout the laser energy delivery to monitor the target and surrounding tissue temperatures. A $15 \mathrm{~W} 980 \mathrm{~nm}$ wavelength diode laser was used to generate the laser light, first as a low-power sub-ablative test pulse to verify position of the heating, and then at ablative intensities. Multiple pulses were generated along the surgical tract to create a contiguous overlapping ablation zone via manual translation of the fiber within the catheter until the entire target was lesioned. A second tract was employed when anatomically necessary in a minority of cases. After post-ablation imaging with various sequences including gadolinium-enhanced T1, the probe was removed. Patients were typically monitored overnight in a standard hospital room, except for a few patients early in the series who were monitored in the intensive care unit for 1 day, and most patients were discharged on postoperative day 1 . Figure 1 presents a series of images outlining the course of the procedure for a representative patient, including preoperative work-up, intraoperative thermal imaging, immediate post-operative imaging and one-year follow-up imaging.

Patients who were not free of disabling seizures following the procedure were considered for further surgical intervention, including open resection and, for patients in whom review of an interval post-operative MRI scan showed a remnant region of the hippocampus and/or uncus thought to be responsible for ongoing seizures, repeat ablations. The latter were performed in a fashion similar to the initial ablation but from a more lateral entry, targeting the remnant medial temporal structures. Patients who failed a repeat ablation were again considered for open surgical procedures.

\section{Outcome Categorization}

All patients had follow-up by a neurosurgeon and/or neurologist typically at 6 weeks, 3 months, 6 months, 12 months and then every 12 months. Assessments included postoperative complications, seizure frequency, antiepileptic medication doses, and quality of life (QOLIE-31) surveys. Surgical outcomes using Engel's classification scheme ${ }^{22}$ were determined by retrospectively analyzing clinic notes and patient correspondence. For patients who underwent additional surgical procedures, such as repeat SLAH or ATL, the last observation prior to the second surgery was carried forward, with the following exception: patients that attained 12 months of outcome following a second SLAH procedure on the same target (i.e. the medial temporal lobe; $n=9$ ) were re-categorized with respect to that second procedure, with the initial procedure having been considered a technical failure. Patients that underwent a subsequent ATL $(n=4)$ continued to be categorized as not seizure free with respect to the previous SLAH. Only patients who had generalized convulsive seizure(s) immediately following antiepileptic drug withdrawal were categorized as Engel $1 \mathrm{D}$, following the original classification scheme. A $95 \%$ confidence interval was generated for the proportion of the patients that remained seizure free for at least 12 months following SLAH.

The postoperative progression of seizure freedom within our cohort was also examined via a Kaplan-Meier analysis, with events being defined as recurrence of debilitating seizures and 
censorship at the point of last follow-up. For those patients having undergone a repeat SLAH the analysis was performed with respect to the repeat procedure.

Quality of life was determined from administration of the QOLIE-31 inventory before SLAH and at one-year follow-up. Only data from patients having QOLIE-31 data before and after SLAH, but before an open resection, if applicable, and having passed performance validity measures, are included. For this inventory, patients' responses were scored on a point scale from $0-100$, with a higher number indicating a more positive outcome in terms of quality of life. Raw scores were then converted to T-scores scaled against the mean of a cohort of epilepsy patients originally used to develop the inventory, with the mean of that original cohort set at 50 . Thus, a score higher than 50 would indicate a more favorable outcome in terms of quality of life than is generally experienced amongst epilepsy patients; a score lower than 50 would indicate a lower quality of life. Overall quality of life was examined through descriptive statistics, as well as through the three subcategories believed to be the most relevant to the outcomes following a surgical intervention: seizure worry, cognitive function, and emotional well-being. A two-tailed repeated measures t-test was performed for the overall quality of life as well as the subcategories.

Verbal memory was assessed using the Rey Auditory Verbal Learning Test (RAVLT), a standard measure of verbal learning and memory used regularly in the context of epilepsy surgery 23,24 . The RAVLT requires free recall of words from a list of 15 unrelated words, which are repeated over five separate trials. Recall is tested at the end of each of 5 trial presentations. An alternative word list is then presented and tested, followed by an immediate recall of the words from the original list. This is followed by another free recall at the end of a 30-minute delay period filled with other unrelated tasks. This test was administered at presurgical baseline and an average of $6.4(\mathrm{SD}=1.5)$ months after surgery (range of 5 to 11 months). We examined the 5-trial learning total and delayed recall scores from the RAVLT, using reliable change index scores ${ }^{25}$. We also used paired sample t-tests to determine whether pre- to post-ablation change was significant at the group level. Forty-nine of the 58 patients had this data available for evaluation.

\section{Results}

\section{Patient Demographics and Diagnostic Data}

A total of 58 patients underwent SLAH, including 29 females and 21 males, ranging in age from 16 to 67 years (mean $40 \pm 15$ years) at the time of first SLAH (Table 1). Thirty patients underwent right-sided and 28 left-sided procedures. MR imaging demonstrated MTS in 43 patients, three of whom had bilateral MTS, which was greater on the operated side. An additional three patients had signal increase in the hippocampus on T2 and/or FLAIR imaging, but lacked hippocampal atrophy. Supplementary Table 1 presents each patient's pre-surgical diagnostic data. Several patients had imaging findings possibly suggestive of secondary epileptogenic foci.

A total of 67 laser ablation procedures were performed: 58 initial procedures and 9 repeat procedures in 9 patients. Two patients with recurrent seizures following SLAH elected to undergo ATL as a secondary procedure, and two additional patients underwent ATL 
following unsuccessful repeat SLAH. Repeat SLAH was performed 12.9 \pm 11.9 months following the initial procedure. The ATLs were performed 8.5 \pm 4.5 months (mean) after the initial or, if performed, repeat SLAH. The mean length of stay was $1.4 \pm 0.7$ days following both initial and $1.3 \pm 0.5$ days following repeat SLAH procedures.

\section{Seizure Outcomes}

Thirty-one of 58 patients (53.4\%, 95\%CI: 40.8\%-65.7\%) were free of disabling seizures (Engel 1) for $\geq 12$ months following SLAH (Figure 2A), including three patients who were initially not seizure free but achieved Engel 1 outcomes for $\geq 12$ months following repeat ablation of remaining medial temporal tissue. Of patients with MTS, 26 of 43 (60.5\%; 95\%CI: $45.6 \%-73.7 \%$ ) were free of disabling seizures at $\geq 12$ months, only one of whom underwent repeat ablation. Conversely, only 5 of 15 (33.3\%; 95\%CI: $15.0 \%-58.5 \%)$ patients without MTS achieved Engel 1 outcomes. Following repeat ablation, 7 of 9 patients experienced improved outcomes, with 3 of those achieving freedom from disabling seizures (Engel 1B) (Figure 2B, C). Of the 31 patients who achieved freedom from disabling seizures, 22 were completely seizure free (Engel 1A), 7 had non-disabling simple partial seizures (Engel 1B), 1 had a single generalized convulsive seizure with antiepileptic drug withdrawal (Engel 1D) and 1 had generalized convulsive seizure associated with hyponatremia secondary to carbamazepine use (Engel 1D).

Four patients underwent ATL following SLAH, two after a single SLAH procedure and two following repeat ablations. After one year, only 1 such patient achieved seizure freedom (a single simple partial seizure occurred at 6 weeks); the others experienced a reduction in seizure frequency (Engel $2[n=1]$ and Engel $3[n=2]$ ). Notably, none of these 4 patients had MTS on MR imaging.

Only 4 of 27 patients not free of disabling seizures underwent open resections. Reasons for not having subsequent open surgery varied, including 1 ) nearly seizure free or only nocturnal seizures $(\mathrm{n}=11) ; 2)$ not interested in further surgery $(\mathrm{n}=6) ; 3)$ moved or lost to follow-up ( $\mathrm{n}=3)$; 4) possible contralateral onsets $(\mathrm{n}=1) ; 5)$ underwent callosotomy $(\mathrm{n}=1)$; and 6) ATL is scheduled to be performed.

\section{Open resections during the study period}

To determine whether there was a selection bias in the SLAH cohort, we tabulated the open temporal lobe resections during the 5-year study period, comprising 22 patients who underwent either ATL $(n=20)$ or SAH $(n=2)$. Reasons varied for undergoing open resection. Six underwent ATLs following failed medial temporal surgeries: 4 SLAH (as noted above), and 2 other medial temporal procedures. Eight ATLs were performed at the completion of invasive monitoring with subdural grid electrodes, along with removal of the electrodes; of these, two had onsets found to be medial temporal. Five patients were offered ATL but not SLAH, for the following reasons: one due to the presence of an ipsilateral visual field deficit from a contralateral occipital stroke (contraindicating an ipsilateral occipital lobe procedure); two due to the presence of other temporal lobe lesions (occipital-temporal gyrus calcified lesion; basal temporal encephalocoele); one due to another possible epileptic focus; and one out of concern for patient inability to return for follow-up (indeed this patient never 
returned for follow-up after surgery). This latter patient, therefore, was the only surgical patient during the timespan of this series that was not offered SLAH despite being a bona fide candidate. Finally, three patients who had been offered both options chose open surgery over SLAH (1 ATL and 2 selective amygdalohippocampectomy). Overall, 11 of 22 (50\%; one lost to follow-up was categorized as not seizure free) were seizure-free (8 Engel 1A, 2 Engel 1B, 1 Engel 1D) at one-year following open temporal surgery. Of these 22, twelve patients could be considered equivalent to the SLAH patients: offered but chose open resection over SLAH ( $n=3)$; only offered ATL due to reasons other than having a possible alternative epileptic zone $(\mathrm{n}=2)$; underwent ATL after invasive monitoring that identified mesial structures as the sole onset zone $(n=2)$; or underwent but failed SLAH $(n=4)$ (i.e. were originally considered SLAH candidates) or another medial temporal procedure $(n=1)$, prior to ATL. Six of these twelve patients (50\%) were seizure-free, all being Engel 1A; 6 of the remaining 10 (i.e. not equivalent) patients who underwent ATL (60\%) were also free of disabling seizures. These data suggest that the results in the SLAH cohort were not due to selection bias, and that these results represent the wide range of patients that present for surgery for MTLE at a high-volume epilepsy center.

\section{Seizure freedom survival analysis}

A Kaplan-Meier analysis of the time until seizure recurrence was performed to determine the long-term efficacy of SLAH. As per the Engel classification scheme, seizures within the first month were excluded. The recurrence rate was greatest within the first six months following SLAH. Seizure recurrence occurred at a lower but steady rate through 24 months (seizure freedom: $34.3 \%$; 95\%CI: $19.7 \%-49.3 \%$ ) after which point seizure recurrence was no longer observed. The longest follow-up without seizure recurrence was 53 months. (Figure 3A). When comparing non-MTS patients to those with MTS, the Mantel-Cox logrank test indicated that there was a significant difference between the seizure-free survival curves (Chi-Square $=6.636, \mathrm{df}=1, \mathrm{p}=0.01$ ) with non-MTS patients being 3.32 (MantelHaenszel hazard ratio; $95 \% \mathrm{CI}: 1.33-8.29)$ times more likely to have seizure recurrence than those with MTS (Figure 3B).

\section{Quality of Life Outcomes}

At the group level, patients ( $n=41)$ who underwent SLAH experienced improved quality of life as compared to their pre-surgical states. Follow-up ranged from 5 to 28 months postSLAH with a mean of $12.4 \pm 4.0$ months. All nine patients who underwent repeat SLAH are included here, with the follow-up outcomes of eight following the repeat SLAH and one following the first SLAH (i.e. before the repeat procedure). For overall quality of life, a significant improvement was observed following SLAH $(\mathrm{p}<0.002)$. There were also significant improvements observed in all of the explored subcategories, including seizure worry $(\mathrm{p}<0.0001)$, cognitive functioning $(\mathrm{p}=0.001)$, and emotional status $(\mathrm{p}<0.05)$.

\section{Complications}

Five visual field deficits (VFD) occurred $(5 / 58=8.6 \%)$, only one of which was persistent and symptomatic (1.7\%) (see Supplemental Table 1). This was a nearly complete homonymous hemianopia that followed a repeat ablation, and may be attributable to thermal spread to the ventral thalamus, in the region of the lateral geniculate nucleus. The second 
VFD was a superior quadrantanopia secondary to a intraparenchymal hematoma in the occipital region; although persistent on formal visual field and confrontation testing, it was asymptomatic on last follow-up. A third patient complained of vague visual difficulties and had a mild incongruous hemianopic central depression, thought to be at the level of the optic tract, but follow-up formal and confrontation visual field testing were normal. The other 2 VFDs were mild superior quadrantanopias that were asymptomatic on follow-up confrontation testing; formal field testing was persistent in one, and not obtained in the other. These mild quadrantanopias are believed to have resulted from the most posterior ablations having encroached upon the optic radiation in the external sagittal stratum. Having recognized this etiology, it did not occur in the last 33 of 58 patients. In addition to the one intraparenchymal hematoma noted, there was one additional hemorrhage, an acute subdural hematoma that was operatively addressed immediately following ablation; it was not associated with a neurologic deficit. It. Four patients $(4 / 58=6.9 \%)$ experienced transient non-disabling partial cranial nerve palsies (III and IV), believed to have resulted from thermal injury spreading medially at the tentorium when ablating the uncus, subiculum and/or entorhinal cortex. All four patients were treated with steroids and recovered completely.

\section{Memory Outcomes}

Four of $49(8.2 \%)$ patients experienced a decline on one $(n=3)$ or both $(n=1)$ sub-scores of the RAVLT (verbal memory) measure. This included 3 of 20 (15\%) patients undergoing SLAH involving their language dominant cerebral hemisphere and 1 of 29 (3.4\%) patients undergoing a nondominant SLAH. Of the 3 language-dominant SLAH patients to significantly decline on this memory measure, two declined on the learning trial only and the third declined on both the learning and delayed recall trials. Notably, two of the patients that declined had dual pathology involving the lateral temporal lobe region (one of these patients also had MTS), and one patient had normal neuroimaging. The single nondominant SLAH patient to experience significant decline involved a patient with right MTS who exhibited a decline on the learning trial only. In contrast, significant improvements were observed in 2 of $20(10 \%)$ patients undergoing language dominant SLAH and 7 of $29(24.1 \%)$ patients undergoing nondominant SLAH. Improvements were typically in the delayed recall score of the RAVLT ( 8 of 9 cases). At the group level, the only significant change following ablation was the improvement seen in delayed verbal recall by the nondominant SLAH group ( $t=$ $-2.08, p<.05)$ (Table 2).

\section{Discussion}

Stereotactic laser amygdalohippocampotomy, a minimally invasive approach to medial temporal lobe surgery using MR-guided laser interstitial thermal therapy, eliminated disabling seizures in 53.4\% (95\%CI 40.8\%- 65.7\%) of all patients with MTLE, and 60.5\% (95\%CI: $45.6 \%-73.7 \%$ ) of patients with MTS, at one-year following surgery. These results mirror and expand our early results ${ }^{19}$ and those of others in smaller uncontrolled cohorts (Table 3). In the aggregate of our series and those currently published series' one-year outcomes, 56 out of 98 patients overall (57.1\%) and 42 out of the 69 patients with MTS $(60.8 \%)$ became free of disabling seizures at one-year follow-up. It should be noted that 
series such as these, describing outcome of a novel approach and representing a center's initial cohort of patients, are prone to be non-representative of a mature technique, reflecting each individual center's 'learning curve' with respect to patient selection and technical performance.

By contrast, there is an extensive experience with both anterior temporal lobectomy and selective amygdalohippocampectomy. Two randomized controlled trials compared ATL to best medical therapy in single-blinded designs. The Canadian trial found that $58 \%$ of patients in the surgical arm ( $\mathrm{N}=40$; intent-to-treat group), and $64 \%$ of operated patients $(\mathrm{N}=36)$, became free of disabling seizures for 1 year following surgery, versus $8 \%$ in the medical $\mathrm{arm}^{2}$. The second, the Early Resective Surgery for Epilepsy Trial (ERSET), found that $73 \%$ of highly-selected operated patients (intent-to-treat group, $\mathrm{N}=15 ; 85 \%$ of patients with a complete data set) remained free of disabling seizures in the second year following surgery, versus no subjects in the control $\mathrm{arm}^{26}$. In contrast to these studies, however, the present cohort represents a 'real-world' series of patients, not a selected subset. This is evident by the paucity of patients in this period who underwent open resection rather than SLAH; that is, we did not exclude patients less likely to experience better outcomes from the treatment. This is reflected in the seizure-free rate amongst the contemporaneous openresection group of 50\%. In contrast, both randomized trials contained criteria that would have excluded many of the patients in our series, even though such patients are in reality treated with temporal lobe surgery in many epilepsy centers and can derive benefits of surgery. For example, whereas $26 \%$ of our patients did not have MTS, only $15 \%$ of patients in the surgical arm in the Canadian trial had "normal" MRI results, and the ERSET trial specifically excluded all such patients.

A more appropriate comparison than RCTs is a recent meta-analysis comparing outcomes following ATL and SAH, comprised of studies less than half of which collected data prospectively ${ }^{3}$. Back calculation of the rates of freedom from disabling seizures from risk ratios and absolute risk reductions derived overall rates of $75 \%$ after ATL and $67 \%$ after SAH. Of note, 1092 of the 1203 patients (91\%) had hippocampal sclerosis, as compared to $75 \%$ in our series, rendering the seizure free rate in that study more comparable to our MTSpositive cohort than our complete cohort. A similar back calculation of seizure freedom rates in the MTS subgroup from the meta-analysis yields a 73\% rate following ATL and 66\% following SAH. By comparison, we observed that $60.5 \%$ of MTS patients treated with SLAH were free of disabling seizures at 12 months. Thus, assuming that the MTS cohorts are comparable among these studies, it appears that ATL is somewhat superior to $\mathrm{SAH}$, and SAH is somewhat superior to SLAH, at least with respect to seizure outcome at 12 months alone.

Only one prior series directly compares outcomes of open temporal lobe surgery with SLAH. While describing select cognitive outcomes, we previously reported similar seizure outcomes following open resection (ATL or SAH) as compared to SLAH ${ }^{18}$. In that series, 39 patients underwent open surgery (all but 3 of them prior to availability of SLAH) and 19 underwent SLAH; there were no significant demographic differences between the cohorts. Engel 1 status at 12 months following surgery was achieved in 24 of $39(62 \%)$ of open surgeries and 11 of 19 (58\%) SLAH patients, comparable to the larger series reported here 
(57\% of 58 patients). In the present study, we analyzed the contemporaneous group of 22 patients undergoing open resections (all but 2 of which were ATL), the outcomes of which (50\% free of disabling seizures) were comparable to the SLAH group (53.4\%), with a not dissimilar number of patients completely seizure free (Engel 1A: $38 \%$ vs. $43 \%$, respectively). However, when we excluded from the analysis of the open-resection group those patients that were not strictly comparable to the patients offered SLAH, while still $50 \%$ were free of disabling seizures, all of those were completely seizure-free (Engel 1A), suggesting that open-resection may be more effective for eliminating auras, which may originate outside of the zone of laser ablation, e.g. within extra-mesial temporal regions. In sum, just as it appears that the chance of becoming free of seizures after SAH is somewhat less than after ATL, a reasonable interpretation of our results and those of others is that there is a further but small reduction of the achievement of seizure freedom after SLAH. More definitive data will require larger prospective trials.

The relative advantages and disadvantages of SLAH versus open resective surgery merit consideration. First and foremost are the comparative surgical risks against which benefits must be weighed. Only one patient (1.7\%) experienced a permanent disabling complication - homonymous hemianopia following repeat SLAH, likely due to thermal injury to the lateral geniculate nucleus. This occurred during the sixth procedure in our series and, with improvements in stereotactic and ablative technique (reflecting learning curve), no similar complications have been seen in 61 subsequent procedures, including 8 other repeat ablations. This complication was reported by another group after de novo SLAH, and the specific anatomical and trajectory-related circumstances contributing to this avoidable complication were discussed ${ }^{27}$. Four other patients in our series experienced visual field deficits, none of which were persistently symptomatic. One resulted from an intraparenchymal hematoma (of which there was only one in the series); and one was a transient and mild injury to the optic tract. The remaining two likely resulted from thermal injury to the optic radiation near the posterior hippocampal body. Recognition of circumstances contributing to this complication (excessive ablation of this region) also makes it avoidable, and none occurred in the final 33 patients. By contrast, in the Canadian RCT one patient (2.8\%) sustained unexpected permanent deficits from a thalamic infarct, and 55\% of patients experienced non-disabling superior quadrantanopias. Again, more definitive risk information awaits larger prospective studies, but it appears that the surgical risks of SLAH are less than those of open procedures, particularly after surmounting the initial technical learning curve.

A second set of risks to consider includes the unintended consequences of open temporal lobe surgery on structures and functions not intrinsic to the seizure onset zone - so called 'collateral damage' 15 . We recently demonstrated that, whereas $88 \%$ of patients following open resection (ATL or SAH) experienced cognitive declines in naming or object recognition, none of our first 19 patients experienced decline in any measure following $\mathrm{SLAH}^{18}$. Furthermore, based on outcome data presented in our current sample, there appears to be less risk of decline in verbal memory following SLAH than after open resection (i.e., $8.2 \%$ of total sample; $15 \%$ of language dominant TLE cases). These numbers are far below the $30-60 \%$ rates of decline reported in the research literature for patients undergoing open resection ${ }^{11,28,29}$. Two of the 3 language dominant TLE cases experiencing 
decline had dual pathology involving their lateral temporal lobe and/or pole and one had normal neuroimaging. These findings should provide caution that verbal memory decline can occur in a minority of SLAH patients, and that specific patient characteristics should be prospectively studied in outcome studies in order to determine the relative risk of decline for the individual patient. Sparing more of what is presumed to be a broad network supporting verbal memory appears to more often contribute to a preserved memory. Overall, these cognitive advantages of SLAH likely derive from avoiding transgression and/or resection of the anterior, lateral, and basal temporal structures. Other groups report preserved naming following language-dominant SLAH ${ }^{27,30-32}$, and, while verbal memory can decline following SLAH, preliminary results from currently published small series hint at better outcomes than those typical of open resection as well $27,30-32$. Thus, on balance, while SLAH may yield a marginal decrease in the chance of freedom from disabling seizures, and possibly the rate of being completely seizure free, as compared to open resection, the cognitive benefits and safety profile of SLAH make it an attractive alternative, especially when considering surgery on the dominant hemisphere. In fact, an analysis taking into consideration the advantages associated with fewer cognitive and other adverse effects suggested that seizure free rates must be greater than $43 \%$ for those advantages to outweigh the disadvantages of a lower rate of seizure freedom ${ }^{33}$. In the present study, SLAH surpasses that threshold: both the actual rate of freedom from disabling seizures, and the lower bound of the confidence interval for those patients with MTS, exceeds $43 \%$.

Third, the socioeconomic advantages and disadvantages of a minimally invasive approach to medial temporal surgery warrant consideration. While socio-economic costs in medical decision-making are complex and take into account numerous factors beyond the scope of this manuscript (e.g. subsequent health care utilization, living independently, rates of return to work, etc.), we note that the mean length of stay after SLAH was 1.4 days, whereas the length of stay for a craniotomy is at minimum two days, and usually longer.

In the foregoing discussion, comparisons were made between laser amygdalohippocampotomy and more invasive open resections, in terms of seizure and neurocognitive outcomes. However, no direct randomized comparison has been made, nor is such a trial feasible in the absence of patient equipoise: many patients and/or care-providers (including referring neurologists) are unwilling to fully consider open temporal lobe surgery. Conventional epilepsy surgery is in fact known to be underutilized for fear of discomfort, disfigurement, surgical risks, and cognitive side-effects. In our own series, several patients conveyed reluctance to undergo open surgery as an alternative to laser ablation, a sentiment still present even in patients with recurrent seizures after SLAH. In fact, only 5 of these patients agreed to undergo open surgery ( 4 have been completed; 1 is scheduled). Six other patients with persistent seizures that would be candidates for further surgery have thus far refused further surgical treatment. This may reflect bias on both the part of patients, who are increasingly aware of the availability of laser ablation for epilepsy, but also on the part of care-givers, including neurologists, neurosurgeons and advanced practice providers. Indeed, in our center we stopped offering open resection as an initial option for patients with left mesial temporal onsets due to the high incidence of decline in naming ability: ${ }^{10,18}$ in the first half of the period of this study, we performed 7 left-sided and 9 right-sided open resections, whereas during the second half we only performed 1 left-sided resection as 
compared to 5 right resections. The advent of FDA-approved neurostimulation has also contributed to the decline in our left-sided open as well as laser ablative procedures ${ }^{34}$. These considerations raise the question of the most appropriate comparator groups for SLAH (and other less-invasive ablative procedures): for patients and practitioners who do not embrace conventional open surgery, the appropriate comparator for ablation is not open surgery but best medical therapy or neurostimulation. Undoubtedly, a seizure free rate of at least 53.4\% is significantly superior to the historical cohorts randomized to best medical management, yielding only $0-8 \%$ chance of prolonged seizure control, as well as to cohorts in studies of hippocampal neurostimulation ${ }^{34-36}$. Yet it remains a concern that patients who elect thermal ablation risk being left with a sub-optimal result if they do not become seizure free and then reject subsequent open surgery, as the foregoing discussion indicates they have a tendency to do.

Stereotactic laser ablation represents a paradigm shift in the surgical management of epilepsy. With careful consideration and technical execution, this generally low-risk highreward approach minimizes recovery time and cognitive risk while still providing a high chance of seizure control. As SLAH presents no barrier to subsequent ablation, open surgery, or other procedures, SLAH now fills an important gap between the "all or none" considerations of continued medical management and open resection. It provides a practical iterative approach to surgical epilepsy when patient need or desire dictates, and its availability improves utilization of potentially curative epilepsy surgery.

\section{Supplementary Material}

Refer to Web version on PubMed Central for supplementary material.

\section{Acknowledgments}

We would like to thank Deqiang Qiu, John Gale, Kelly Bijanki and our colleagues from the Emory University Biomedical Imaging Technology Center (BITC) for their support of our research, including Xiaoping Hu and Robert Smith, III. Likewise, this research is indebted to Dr. Drane's lab coordinator, Gloria Novak, and research assistants, Ana-Maria Eisner, Michele Price, Amanda Eakin, and Hanna Pellisier. This project was in part supported by a research grant from Visualase, Inc. [Agency Award \#: VIS-10-001] and grants received by DLD from the National Institute of Neurological Disorders and Stroke (NINDS) of the National Institutes of Health (NIH) [K23 NSO49100, K02NS070960, R01NS088748], and Medtronic, Inc. [A1225797BFN:1056035].

\section{References}

1. Hauser WA, Kurland LT. Epidemiology of epilepsy in Rochester, Minnesota, 1935 through 1967. Epilepsia. 1975; 16(1):1-66. [PubMed: 804401]

2. Wiebe S, Blume WT, Girvin JP, Eliasziw M, Effectiveness Efficiency Surgery T. A randomized, controlled trial of surgery for temporal-lobe epilepsy. New England Journal of Medicine. 2001 Aug 2; 345(5):311-8. [PubMed: 11484687]

3. Josephson CB, Dykeman J, Fiest KM, et al. Systematic review and meta-analysis of standard vs selective temporal lobe epilepsy surgery. Neurology. 2013 Apr; 80(18):1669-76. [PubMed: 23553475]

4. Seidenberg M, Hermann B, Wyler AR, Davies K, Dohan FC, Leveroni C. Neuropsychological outcome following anterior temporal lobectomy in patients with and without the syndrome of mesial temporal lobe epilepsy. Neuropsychology. 1998 Apr; 12(2):303-16. [PubMed: 9556776]

5. Spencer S, Huh L. Outcomes of epilepsy surgery in adults and children. Lancet Neurology. 2008 Jun; 7(6):525-37. [PubMed: 18485316] 
6. Helmstaedter C. Temporal lobe resection-does the prospect of seizure freedom outweigh the cognitive risks? Nat Clin Pract Neurol. 2008 Feb; 4(2):66-7. [PubMed: 17955042]

7. Crane J, Milner B. Do I know you? Face perception and memory in patients with selective amygdalo-hippocampectomy. Neuropsychologia. 2002; 40(5):530-8. [PubMed: 11749983]

8. Drane DL, Ojemann GA, Aylward E, et al. Category-specific naming and recognition deficits in temporal lobe epilepsy surgical patients. Neuropsychologia. 2008; 46(5):1242-55. [PubMed: 18206185]

9. Drane DL, Ojemann JG, Phatak V, et al. Famous face identification in temporal lobe epilepsy: Support for a multimodal integration model of semantic memory. Cortex. 2013 Jun; 49(6):1648-67. [PubMed: 23040175]

10. Busch RM, Floden DP, Prayson B, et al. Estimating risk of word-finding problems in adults undergoing epilepsy surgery. Neurology. 2016 Nov; 87(22):2363-9. [PubMed: 27815406]

11. Baxendale S, Thompson PJ, Sander JW. Neuropsychological outcomes in epilepsy surgery patients with unilateral hippocampal sclerosis and good preoperative memory function. Epilepsia. 2013 Sep; 54(9):e131-e4. [PubMed: 23875960]

12. Lutz MT, Clusmann H, Elger CE, Schramm J, Helmstaedter C. Neuropsychological outcome after selective amygdalohippocampectomy with transsylvian versus transcortical approach: A randomized prospective clinical trial of surgery for temporal lobe epilepsy. Epilepsia. $2004 \mathrm{Jul}$; 45(7):809-16. [PubMed: 15230706]

13. von Rhein B, Nelles M, Urbach H, Von Lehe M, Schramm J, Helmstaedter C. Neuropsychological outcome after selective amygdalohippocampectomy: subtemporal versus transsylvian approach. Journal of Neurology Neurosurgery and Psychiatry. 2012 Sep; 83(9):887-93.

14. Gleissner U, Helmstaedter C, Schramm J, Elger CE. Memory outcome after selective amygdalohippocampectomy: A study in 140 patients with temporal lobe epilepsy. Epilepsia. 2002 Jan; 43(1):87-95. [PubMed: 11879392]

15. Helmstaedter C, Richter S, Roeske S, Oltmanns F, Schramm J, Lehmann T-N. Differential effects of temporal pole resection with amygdalohippocampectomy versus selective amygdalohippocampectomy on material-specific memory in patients with mesial temporal lobe epilepsy. Epilepsia. 2008 Jan; 49(1):88-97. [PubMed: 17941848]

16. Curry DJ, Gowda A, McNichols RJ, Wilfong AA. MR-guided stereotactic laser ablation of epileptogenic foci in children. Epilepsy \& Behavior. 2012 Aug; 24(4):408-14. [PubMed: 22687387]

17. McCracken DJ, Willie JT, Fernald BA, et al. Magnetic Resonance Thermometry-Guided Stereotactic Laser Ablation of Cavernous Malformations in Drug-Resistant Epilepsy: Imaging and Clinical Results. Operative Neurosurgery. 2016 Mar; 12(1):39-48. [PubMed: 27959970]

18. Drane DL, Loring DW, Voets NL, et al. Better object recognition and naming outcome with MRIguided stereotactic laser amygdalohippocampotomy for temporal lobe epilepsy. Epilepsia. 2015 Jan; 56(1):101-13. [PubMed: 25489630]

19. Willie JT, Laxpati NG, Drane DL, et al. Real-Time Magnetic Resonance-Guided Stereotactic Laser Amygdalohippocampotomy for Mesial Temporal Lobe Epilepsy. Neurosurgery. 2014 Jun; 74(6): 569-84. discussion 84-5. [PubMed: 24618797]

20. Willie, JT., Tung, JK., Gross, RE. Chapter 16 - MRI-Guided Stereotactic Laser Ablation A2 Golby, Alexandra J. Image-Guided Neurosurgery. Boston: Academic Press; 2015. p. 375-403.

21. Gross RE, Willie JT, Drane DL. The Role of Stereotactic Laser Amygdalohippocampotomy in Mesial Temporal Lobe Epilepsy. Neurosurgery Clinics of North America. 2016 Jan; 27(1):37-50. [PubMed: 26615106]

22. Engel, J., Van Ness, PC., Rasmussen, TB., Ojemann, LM. Outcome with Respect to Epileptic Seizures. In: Engel, J., editor. Surgical Treatment of the Epilepsies. Second. New York: Raven Press; 1993. p. 609-21.

23. Rey A. L'examen psychologique dans les cas d'encephalopathie traumatique. Archives de Psychologie. 1941; 28:286-340.

24. Loring DW, Lowenstein DH, Barbaro NM, et al. Common data elements in epilepsy research: Development and implementation of the NINDS epilepsy CDE project. Epilepsia. 2011 Jun; 52(6): 1186-91. [PubMed: 21426327] 
25. Sawrie SM, Chelune GJ, Naugle RI, Luders HO. Empirical methods for assessing meaningful neuropsychological change following epilepsy surgery. J Int Neuropsychol Soc. 1996 Nov; 2(6): 556-64. [PubMed: 9375160]

26. Engel J, McDermott MP, Wiebe S, et al. Early Surgical Therapy for Drug-Resistant Temporal Lobe Epilepsy A Randomized Trial. Jama-Journal of the American Medical Association. 2012 Mar; 307(9):922-30.

27. Jermakowicz WJ, Kanner AM, Sur S, et al. Laser thermal ablation for mesiotemporal epilepsy: Analysis of ablation volumes and trajectories. Epilepsia. 2017 Feb 28.

28. Helmstaedter C. Cognitive outcomes of different surgical approaches in temporal lobe epilepsy. Epileptic Disorders. 2013 Sep; 15(3):221-39. [PubMed: 23899718]

29. Hill SW, Gale SD, Pearson C, Smith K. Neuropsychological outcome following minimal access subtemporal selective amygdalohippocampectomy. Seizure-European Journal of Epilepsy. 2012 Jun; 21(5):353-60. [PubMed: 22459316]

30. Kang JY, Wu C, Tracy J, et al. Laser interstitial thermal therapy for medically intractable mesial temporal lobe epilepsy. Epilepsia. 2016 Feb; 57(2):325-34. [PubMed: 26697969]

31. Waseem H, Osborn KE, Schoenberg MR, et al. Laser ablation therapy: An alternative treatment for medically resistant mesial temporal lobe epilepsy after age 50. Epilepsy \& Behavior. 2015 Oct. 51:152-7. [PubMed: 26280814]

32. Dredla BK, Lucas JA, Wharen RE, Tatum WO. Neurocognitive outcome following stereotactic laser ablation in two patients with MRI-/PET plus mTLE. Epilepsy \& Behavior. 2016 Mar.56:447. [PubMed: 26828690]

33. Attiah MA, Paulo DL, Danish SF, Stein SC, Mani R. Anterior temporal lobectomy compared with laser thermal hippocampectomy for mesial temporal epilepsy: A threshold analysis study. Epilepsy Research. 2015 Sep.115:1-7. [PubMed: 26220371]

34. Morrell MJ. Responsive cortical stimulation for the treatment of medically intractable partial epilepsy. Neurology. 2011 Sep 27; 77(13):1295-304. [PubMed: 21917777]

35. Heck CN, King-Stephens D, Massey AD, et al. Two-year seizure reduction in adults with medically intractable partial onset epilepsy treated with responsive neurostimulation: Final results of the RNS System Pivotal trial. Epilepsia. 2014 Mar; 55(3):432-41. [PubMed: 24621228]

36. Bergey GK, Morrell MJ, Mizrahi EM, et al. Long-term treatment with responsive brain stimulation in adults with refractory partial seizures. Neurology. 2015 Feb 24; 84(8):810-7. [PubMed: 25616485] 

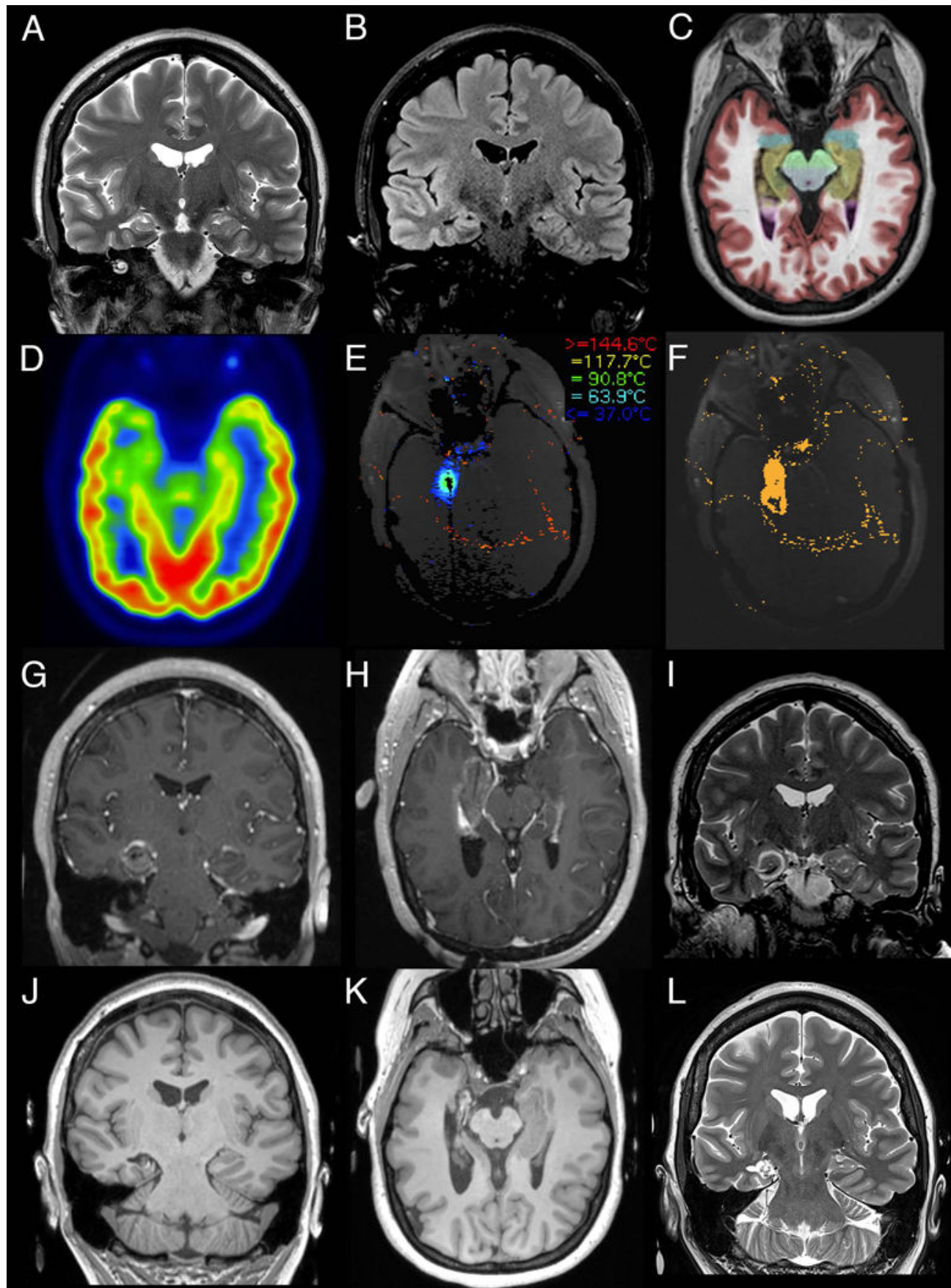

Figure 1. Representative MR images from the pre- and post-operative course of a representative SLAH patient with right MTS

Pre-ablative diagnostic workup demonstrates the characteristic findings of MTS being hippocampal atrophy with increased T2 (A, coronal) and T2-FLAIR (B, coronal) intensity. This is further highlighted by NeuroQuant (CorTechs Laboratory, San Diego, CA) segmentation (C, axial) with the colorized medial temporal lobe structures (hippocampus in brown and amygdala in blue) overlaid on T1 imaging. Pre-operative FDG-PET (D, axial) demonstrates hypometabolism in the right medial temporal structures, consistent with right MTS. Intraoperative MR thermometry is utilized to guide the procedure (E, axial screenshot 
of Visualase thermal map generated during LITT of the amygdala) and assess the thermal damage following the procedure ( $\mathrm{F}$, axial screenshot of Visualase irreversible damage estimation). Immediate post-ablative imaging demonstrates $\mathrm{T} 1$ hypointensity of the ablation target with peripheral contrast enhancement (G, coronal and H, axial, MPRAGE) and T2 (I, coronal) hypointense rings surrounding the ablation. 12-month follow-up imaging demonstrates necrosis and volume reduction of the target tissue with the resulting cavitation on T1 (J, coronal and $\mathrm{K}$, axial MPRAGE) and T2 hyperintensity (L, coronal). 


\section{A Engel Class at 12 Months Following SLAH}
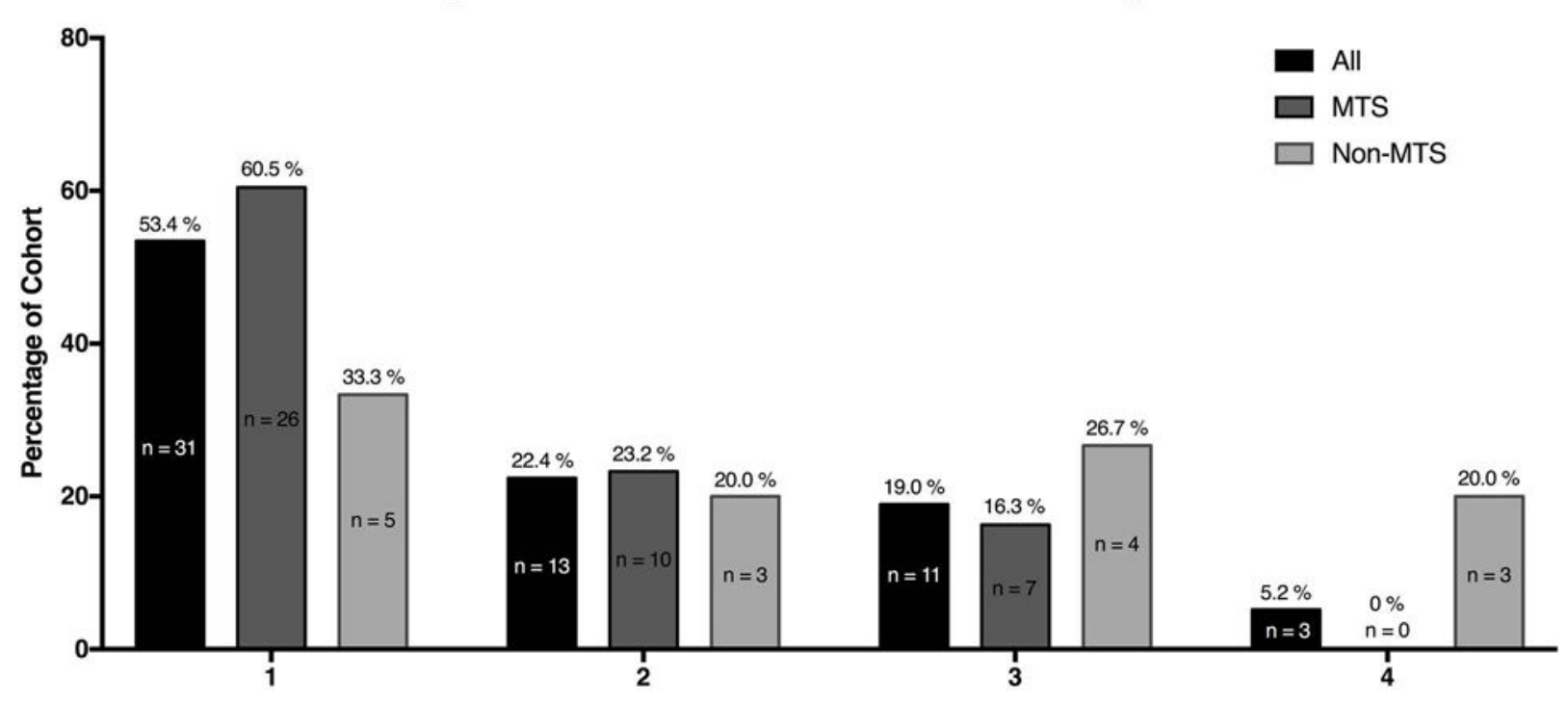

$B$

Engel Class

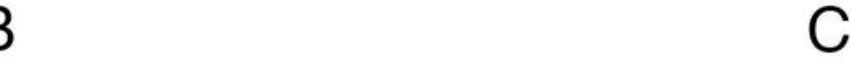

Seizure Freedom Outcomes
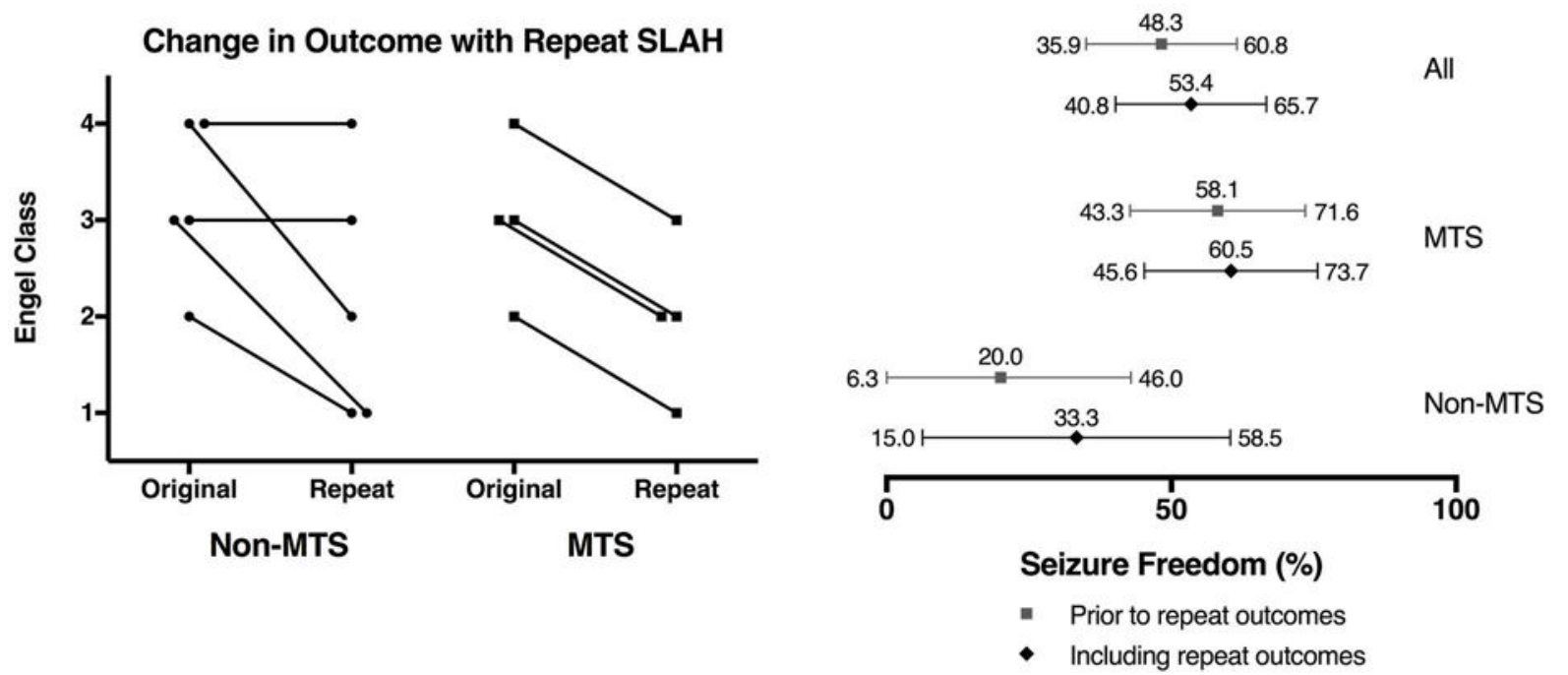

Figure 2. Outcome following SLAH at 12-month follow-up

(A) Distribution of 12-month patient outcomes by Engel class with respect to last SLAH procedure. The numbers above the bars indicate the percentage of patients in each class and the numbers within the bars equal the number of patients (Bar color: All: Black; MTS: Dark Grey; Non-MTS: Light Grey). (B) Comparison of 12-month patient outcomes as stratified by Engel class for patients having undergone repeat SLAH with the 12-month outcomes following the first SLAH (Original) compared with the 12-month outcome following the repeat SLAH (Repeat) demonstrating sustained or improved outcome following the repeat procedure. Non-MTS and MTS groups are presented. (C) The one-year seizure freedom rates observed for all patients with the $95 \%$ CIs. The black diamonds are the outcomes with respect to the last SLAH procedure (i.e. including repeat procedures) and the grey squares 
are the outcomes with respect to only the first procedure. All patients are presented, as well as the sub groups of MTS and Non-MTS. 
A

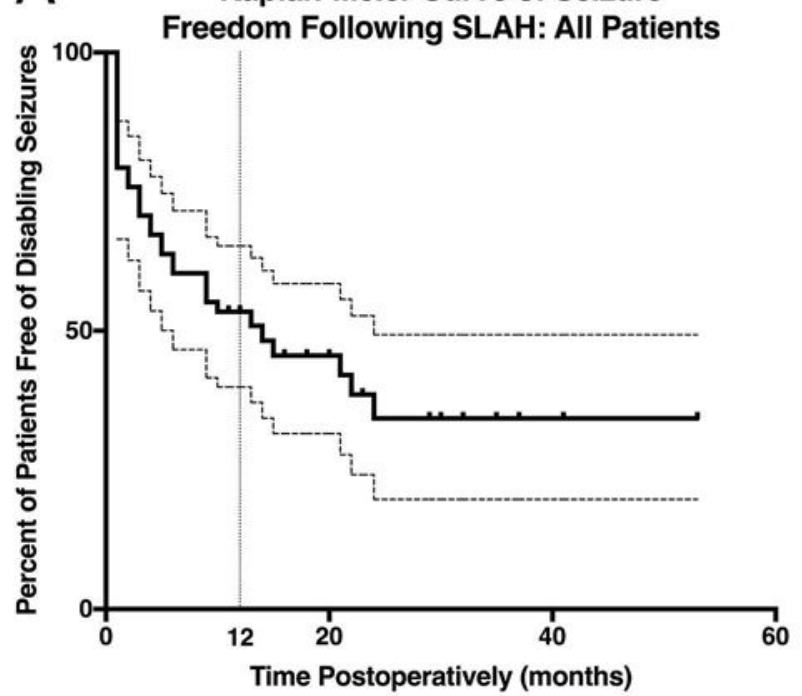

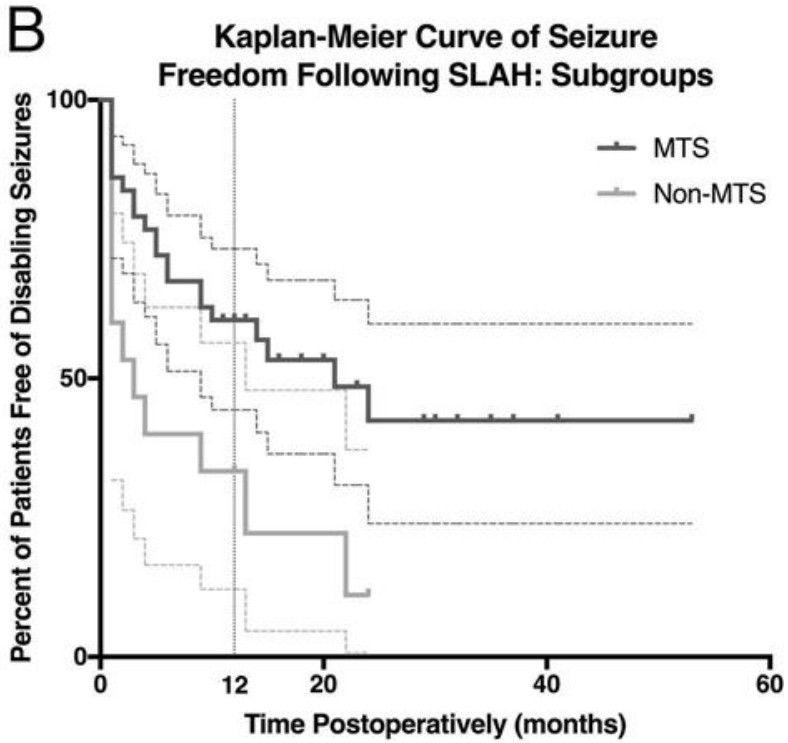

Figure 3. Long term seizure outcomes

Kaplan-Meier depiction of the time to recurrent seizures following last SLAH. All patients (A) are presented, as well as a comparison (B) of the patients with MTS (dark grey) to those without (light grey). Dashed lines corresponding in color to their solid line counterparts indicate 95\% confidence intervals. Tick marks indicate censorship. A vertical grey dashed line is presented to indicate the 12-month time point. 


\section{Table 1}

\section{Demographics}

\begin{tabular}{|c|c|}
\hline Patient Demographics & \\
\hline Female $(\%)$ & $56.9(\mathrm{n}=33)$ \\
\hline \multicolumn{2}{|l|}{ Age (y; mean \pm standard deviation) } \\
\hline At SLAH & $40.4 \pm 15.1$ \\
\hline At First Non-Febrile Seizure & $17.1 \pm 14.2$ \\
\hline Left-Sided Procedures (\%) & $48.3(n=28)$ \\
\hline \multicolumn{2}{|l|}{ Radiological Findings (\%) } \\
\hline Unilateral MTS only & $48.3(n=28)$ \\
\hline Other ${ }^{*}$ & $6.9(n=4)$ \\
\hline Both MTS and Other ${ }^{*}$ & $25.8(\mathrm{n}=15)$ \\
\hline Normal & $19.0(\mathrm{n}=11)$ \\
\hline Prior Surgery for Epilepsy $(\%)^{* * *}$ & $5.2(\mathrm{n}=3)$ \\
\hline
\end{tabular}

* Other radiological findings included temporal lobe-grey white matter blurring, dysmorphic temporal lobe, dysmorphic hippocampi, temporal asymmetry, hippocampal asymmetry, diffuse atrophy (temporal and parietal), atrophic fornix and mammillary body, encephalomalacia (frontal periventricular, temporal and occipital), gliosis, parietal arachnoid cyst, parietal calvarium lesion, subependymal grey matter heterotopia (periventricular and frontal), bifrontal contusions, temporal bone fracture, microhemorrhages (corpus callosum, thalamus, frontal cortex and subcortex), microvascular ischemic changes, resection of parieto-occipital meningioma and traumatic brain injury.

***

Surgeries included an anterior-two-thirds corpus callosotomy, an amygdalohippocampectomy and resection of a temporal juvenile pilocytic astrocytoma. None of these occurred in patients with unilateral MTS only or MRI normal patients. Two of these surgeries were performed in patients with MTS plus other radiological findings.

Abbreviations: SLAH, stereotactic amygdalohippocampotomy; MTS, mesial temporal sclerosis 


\section{Table 2}

Verbal memory outcomes

\begin{tabular}{|l|c|c|c|c|}
\hline & \multicolumn{2}{|c|}{ RAVLT- Learning } & \multicolumn{2}{c|}{ RAVLT-Delayed Recall } \\
\cline { 2 - 5 } & Pre SLAH & Post SLAH & Pre SLAH & Post SLAH \\
\hline $\begin{array}{l}\text { All Subjects } \\
(\mathrm{n}=49)\end{array}$ & $\begin{array}{c}41.8 \pm 10.8 \\
(14-65)\end{array}$ & $\begin{array}{c}41.9 \pm 11.6 \\
(11-59)\end{array}$ & $\begin{array}{c}5.9 \pm 3.9 \\
(0-15)\end{array}$ & $\begin{array}{c}6.5 \pm 4.1 \\
(0-14)\end{array}$ \\
\hline $\begin{array}{l}\text { Language Dominant Hemisphere SLAH } \\
\text { (n=20) }\end{array}$ & $\begin{array}{c}37.4 \pm 10.7 \\
(14-62)\end{array}$ & $\begin{array}{c}35.3 \pm 12.7 \\
(11-56)\end{array}$ & $\begin{array}{c}4.6 \pm 3.7 \\
(0-13)\end{array}$ & $\begin{array}{c}4.2 \pm 3.4 \\
(1-12)\end{array}$ \\
\hline $\begin{array}{l}\text { Non-dominant Hemisphere SLAH } \\
(\mathrm{n}=29)\end{array}$ & $\begin{array}{c}44.9 \pm 10.0 \\
(33-65)\end{array}$ & $\begin{array}{c}46.6 \pm 8.3 \\
(22-59)\end{array}$ & $\begin{array}{c}6.6^{*} \pm 3.9 \\
(1-15)\end{array}$ & $\begin{array}{c}8.2^{*} \pm 3.7 \\
(0-14)\end{array}$ \\
\hline
\end{tabular}

Note: Scores presented as Mean \pm Standard Deviation (Range)

Scores differed significantly across pre- and post-surgical ablation time points $(p<.05)$.

Abbreviations: RAVLT, Rey Auditory Verbal Learning Test; SLAH, stereotactic laser amygdalohippocampotomy. 

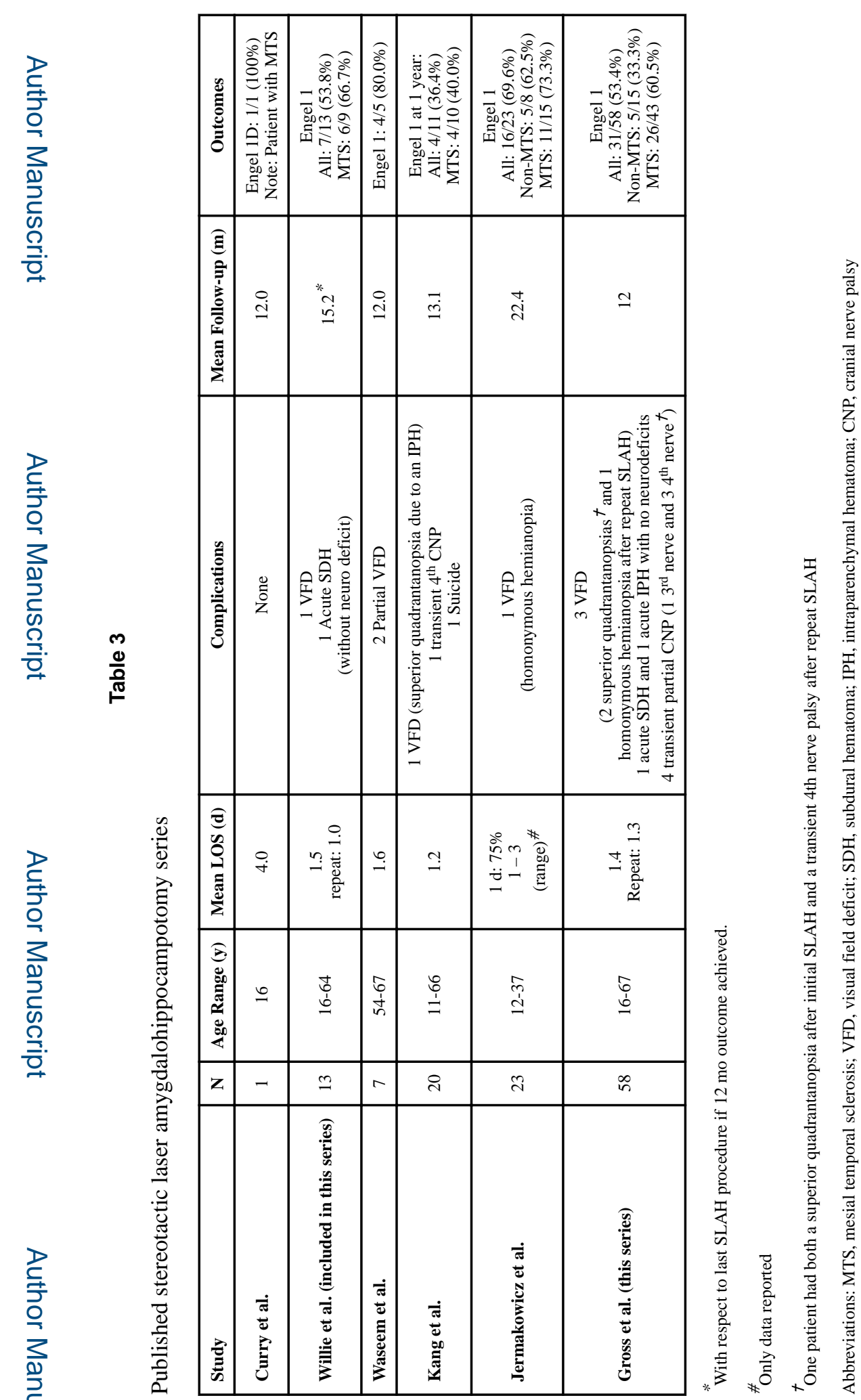

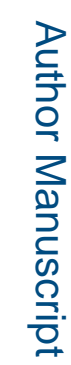

